\title{
First-principles approach for effective elastic-moduli calculation: Application to continuous fractal structure
}

\author{
Ping Sheng \\ Corporate Research Science Laboratories, Exxon Research and Engineering Company, \\ Clinton Township, Annandale, New Jersey 08801 \\ Ruibao Tao* \\ Department of Physics, San Francisco State University, \\ San Francisco, California 94132 \\ (Received 4 February 1985)
}

\begin{abstract}
We present a new first-principles approach to the calculation of effective elastic moduli of composites based on the iterative solution of the Dyson equation for elastic wave scatterings in inhomogeneous media. Application of the method to the examination of elastic scaling behavior of a continuous fractal shows that the moduli associated with different elastic eigenmodes exhibit distinct exponents in scaling with the size of the system.
\end{abstract}

Recently, the distinction between the electrical and the elastic percolation behaviors has been the subject of several studies using discrete elastic models of mass points linked by springs. ${ }^{1-3}$ While the primary focus of these discrete models is to simulate the behavior of percolation clusters, their consideration has also raised interest in regard to the elastic properties of composites in general and the elastic scaling behaviors in particular. However, together with the interest comes the realization that the calculation of elastic moduli for continuous composite with more than a simple microstructure is a difficult task due to the complexity of the associated boundary value problem. This accounts for the scarcity of first-principles calculations on continuous elastic models. In this work we present a new approach to the calculation of elastic moduli for continuous composites. The new method incorporates the boundary conditions implicitly in the equation of motion, thereby circumventing the traditional difficlty of matching boundary conditions across complex interfaces. As an application of the approach, we use it to examine a question in the general nature of elastic scaling properties. That is, for a given continuous fractal structure, do all the independent elastic moduli always scale with the same exponent as automatically assumed previously? Our calculation on a continuous Sierpinski carpet ${ }^{4}$ shows that the moduli associated with different elastic eigenmodes exhibit distinct exponents in scaling with the size of the system. This result, which was not observed in previous discrete models, suggests that not only can there be more than one elastic scaling behavior for a given structure, but also the number of distinct scaling exponents may be dependent on the symmetry of the system. In the following, the formulation of the first-principles approach will be presented in the context of the Sierpinski carpet calculaton.

In Fig. 1 we show the three stages of a Sierpinski carpet made of locally isotropic solid characterized by the two Lame constants $\lambda$ and $\mu$. The size of the system $L$ is defined to be the ratio between the sides of the outer square to the smallest square. For each stage, the unit is repeated periodically in both directions. To calculate the three elastic constants $\bar{K}_{11}, \bar{K}_{12}$, and $\bar{K}_{44}$ of the structure, consider the inhomogeneous elastic-wave equation ${ }^{5}$

$$
\rho(\mathbf{r}) \frac{\partial^{2} \mathbf{u}}{\partial t}=[\lambda(\mathbf{r})+\mu(\mathbf{r})] \nabla(\nabla \cdot \mathbf{u})+\mu(\mathbf{r}) \nabla^{2} \mathbf{u}+[(\nabla \mathbf{u}) \cdot \nabla \lambda(\mathbf{r})+\nabla \mu(\mathbf{r}) \cdot(\nabla \mathbf{u})+(\nabla \mathbf{u}) \cdot \nabla \mu(\mathbf{r})],
$$

where $\rho(r)$ is the density, $u(r)$ is the displacement field, and $\lambda(r), \mu(r)$ are the spatially varying elastic constants. For an abrupt material interface, $\lambda(\mathbf{r})$ and $\mu(\mathbf{r})$ approach a step function. The classical elasticity boundary conditions, displacements, and tractions continuous across the interface, can be shown to result directly from Eq. (1). If follows that the solution of Eq. (1) should yield complete dynamical information about the system. For the calculation of effective moduli (which are defined in the long-wavelength limit) of a periodic system, it is much simpler to deal with the Fourier transform of Eq. (1):

$$
\sum_{\beta}\left[E^{2} \delta_{\alpha \beta}-\omega_{\alpha \beta}^{2}(\mathbf{k})\right] u_{\beta}(\mathbf{k})=\sum_{\mathbf{K}_{n} \neq 0}\left(\sum_{\beta} \frac{1}{\rho(0)} V_{\alpha \beta}\left(\mathbf{k}, \mathbf{K}_{n}\right) u_{\beta}\left(\mathbf{k}-\mathbf{K}_{n}\right)-E^{2} \frac{\rho\left(\mathbf{K}_{n}\right)}{\rho(0)} u_{\alpha}\left(\mathbf{k}-\mathbf{K}_{n}\right)\right) .
$$

Here we have assumed the time dependence of $\mathbf{u}$ is $\exp (-i E t), \alpha, \beta=1,2$, and 3 denote the three components of a vector, $\mathbf{k}$ is a continuous wave vector, $\mathbf{K}_{n}$ is the $n$th reciprocal lattice vector of the periodic structure (for three-dimensional structures $n \equiv\left\{n_{1}, n_{2}, n_{3}\right\}$, where the three numbers index the periodicities along the three principal axes), and

$$
\begin{aligned}
& V_{\alpha \beta}\left(\mathbf{k}, \mathbf{K}_{n}\right)=\left\{\begin{array}{l}
{\left[\lambda\left(\mathbf{K}_{n}\right)+\mu\left(\mathbf{K}_{n}\right)\right] k_{\alpha}\left(k_{\alpha}-K_{n \alpha}\right)+\mu\left(\mathbf{K}_{n}\right) \mathbf{k} \cdot\left(\mathbf{k}-\mathbf{K}_{n}\right), \quad \alpha=\beta,} \\
\lambda\left(\mathbf{K}_{n}\right) k_{\alpha}\left(k_{\beta}-K_{n \beta}\right)+\mu\left(\mathbf{K}_{n}\right) k_{\beta}\left(k_{\alpha}-K_{n \alpha}\right), \quad \alpha \neq \beta,
\end{array}\right. \\
& \omega_{\alpha \beta}^{2}(\mathbf{k})=\frac{1}{\rho(0)} V_{\alpha \beta}(\mathbf{k}, 0) .
\end{aligned}
$$




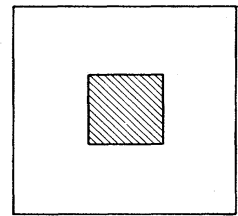

1

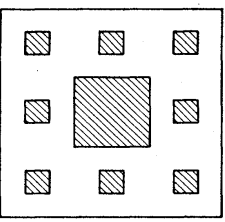

2

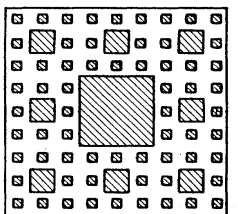

3
FIG. 1. The three stages of a Sierpinski carpet. Area that is empty is indicated by shading. The ratio between the sides of successively sized squares is 3 .

It should be remarked that $V_{\alpha \beta}\left(\mathbf{k}, \mathbf{K}_{n}\right), \mathbf{K}_{n} \neq 0$, simply represents the effect of multiple scatterings by the elastic constant inhomogeneities. For a homogeneous medium, the right-hand side of Eq. (3) vanishes, and it is straightforward to check that the diagonalization of the left-hand side directly yields the eigenfrequencies of acoustic and shear waves.

If we now specialize to the case of elastic waves in the limit of $|\mathbf{k}| \rightarrow 0$, with $E \alpha|\mathbf{k}|$, then an analysis of the order of magnitude of the various terms show that the

$$
E^{2} \rho\left(\mathbf{K}_{n}\right) u_{\alpha}\left(\mathbf{k}-\mathbf{K}_{n}\right) / \rho(0) \quad\left(\mathbf{K}_{n} \neq 0\right)
$$

term is of higher order in $|\mathbf{k}|$ than the other terms and therefore can be neglected in the calculation of effective moduli. Physically, the absence of density effects [apart from $\rho(0)]$ is expected since the effective moduli measures only the static potential energy of the system, which is in- dependent of $\rho$. If now for $\mathbf{K}_{n} \neq 0$ we define a scattering matrix $\hat{S}\left(\mathbf{k}, \mathbf{K}_{n}\right)$ linking $\mathbf{u}\left(\mathbf{k}-\mathbf{K}_{n}\right)$ to $\mathbf{u}(\mathbf{k})$ such that

$$
\mathbf{u}\left(\mathbf{k}-\mathbf{K}_{n}\right)=\hat{S}\left(\mathbf{k}, \mathbf{K}_{n}\right) \mathbf{u}(\mathbf{k}) \text {, }
$$

then Eq. (2) becomes

$$
\sum_{\beta}\left[v^{2} \delta_{\alpha \beta}-C_{\alpha \beta}^{2}(\mathbf{k})-\tau_{\alpha \beta}(\mathbf{k})\right] u_{\beta}(\mathbf{k})=0,
$$

where $v^{2} \equiv E^{2} /|\mathbf{k}|^{2}, C_{\alpha \beta}^{2}(\mathbf{k})=\omega_{\alpha \beta}^{2}(\mathbf{k}) /|\mathbf{k}|^{2}$, and

$$
\tau_{\alpha \beta}(\mathbf{k})=\frac{1}{\rho(0)} \sum_{\mathbf{K}_{n} \neq 0} \sum_{\eta}|\mathbf{k}|^{-2} V_{\alpha \eta}\left(\mathbf{k}, \mathbf{K}_{n}\right) S_{\eta \beta}\left(\mathbf{k}, \mathbf{K}_{n}\right) \text {. }
$$

Diagonalization of Eq. (6) yields directly the eigenvelocities $v$ of the inhomogeneous structure. The analysis of the associated eigenfunctions could then tell us about the polarizations of the elastic-wave eigenmodes. For a longtitudinal wave in a cubic structure $[\mathbf{k} \|(1,0,0)]$, for example, we can define $\bar{K}_{11}=v^{2} \rho(0)$. Other moduli can be defined similarly.

From the definition of $\hat{S}\left(\mathbf{k}, \mathbf{K}_{n}\right)$, Eq. (5), it can be shown after straightforward but lengthy algebra that the scattering matrix $\hat{S}$ rigorously satisfies the matrix Dyson's equation for elastic wave scattering:

$$
\begin{aligned}
\hat{S}\left(\mathbf{k}, \mathbf{K}_{n}\right)= & \hat{F}\left(\mathbf{k}-\mathbf{K}_{n}, \mathbf{K}_{n}\right) \\
& +\sum_{\substack{\mathbf{K}_{n}^{\prime} \neq \mathbf{K}_{n} \\
\neq 0}} \hat{F}\left(\mathbf{k}-\mathbf{K}_{n}, \mathbf{K}_{n}-\mathbf{K}_{n}^{\prime}\right) \hat{S}\left(\mathbf{k}, \mathbf{K}_{n}^{\prime}\right) .
\end{aligned}
$$

Here the elements of $\hat{F}$ are defined by

$$
\begin{aligned}
F_{\alpha \beta}\left(\mathbf{k}-\mathbf{K}_{n}, \mathbf{K}_{n}-\mathbf{K}_{n}^{\prime}\right)= & \frac{1}{1+\gamma_{\alpha}\left(\mathbf{k}-\mathbf{K}_{n}\right)} \frac{1}{E^{2}-\omega_{\alpha \alpha}^{2}\left(\mathbf{k}-\mathbf{K}_{n}\right)} \\
& \times\left(V_{\alpha \beta}\left(-\mathbf{k}+\mathbf{K}_{n}, \mathbf{K}_{n}-\mathbf{K}_{n}^{\prime}\right)+A \frac{\left(\mathbf{k}-\mathbf{K}_{n}\right)_{\alpha}}{1-\delta\left(\mathbf{k}-\mathbf{K}_{n}\right)} \sum_{\eta} \frac{\left(\mathbf{k}-\mathbf{K}_{n}\right)_{\eta}}{1+\gamma_{\eta}\left(\mathbf{k}-\mathbf{K}_{n}\right)} \frac{V_{\eta \beta}\left(-\mathbf{k}+\mathbf{K}_{n}, \mathbf{K}_{n}-\mathbf{K}_{n}^{\prime}\right)}{E^{2}-\omega_{\eta \eta}^{2}\left(\mathbf{k}-\mathbf{K}_{n}\right)}\right),
\end{aligned}
$$

where $A=[\lambda(0)+\mu(0)] / \rho(0)$,

$$
\gamma_{\alpha}\left(\mathbf{k}-\mathbf{K}_{n}\right)=A \frac{\left(\mathbf{k}-\mathbf{K}_{n}\right)_{\alpha}^{2}}{E^{2}-\omega_{\alpha \alpha}^{2}\left(\mathbf{k}-\mathbf{K}_{n}\right)}
$$

and

$$
\delta\left(\mathbf{k}-\mathbf{K}_{n}\right)=\sum_{\alpha} \frac{\gamma_{\alpha}\left(\mathbf{k}-\mathbf{K}_{n}\right)}{1+\gamma_{\alpha}\left(\mathbf{k}-\mathbf{K}_{n}\right)} .
$$

Equation (8), with $E^{2}$ neglected in comparison to $\omega_{\alpha \alpha}^{2}\left(\mathbf{k}-\mathbf{K}_{n}\right)\left(\mathbf{K}_{n} \neq 0\right)$, can be solved iteratively. Rapid numerical convergence was observed. In the worst case (spheres just touching, for example) convergence to three or four singinficant figures was obtained after 15-20 iterations. Physically, the iterations can be interpreted as multiple scatterings of the plane wave by structural inhomogeneities.

The combination of using Fourier coefficients of material parameters as inputs and the capacity to harness the iterative solution technique makes the approach described above generally applicable to the elastic-moduli calculation of periodic composites with arbitrary unit-cell geometry. We have successfully used this method to evaluate the moduli of three-dimensional, two-component composites and biconnected porous frames. ${ }^{6}$ For our present application, Eq. (8) is solved for up to a maximum $\pm N$ of $K_{n}$ 's for each of the two directions, with $\lambda+2 \mu=1$ and $\mu=0.4$. The convergence of the solution is monitored as a function of $N$. In Fig. 2(a) we plot the $\bar{K}_{11} / \rho(0)$ for stages 1,2 , and 3 as a function of $1 / N$. The fact that the points lie on good straight lines is utilized to extrapolate the results to $1 / N=0$. In Fig. 2(b) we show $\log _{10}[\bar{K} / \rho(0)]$ plotted as a function of $\log _{10} L$. From the excellent power-law behaviors we deduce that $\bar{K}_{i} / \rho(0) \propto L^{-\alpha_{i}}$, where $\bar{K}_{1} \equiv \bar{K}_{11}$ [longitudinal wave in (10) direction], $\bar{K}_{2} \equiv \bar{K}_{11}-\bar{K}_{12}$ [shear wave in (11) direction], and $\bar{K}_{3} \equiv \bar{K}_{44}$ [shear wave in (10) direction] are the three moduli associated with three of the eigenmodes of elastic-wave excitations. The values of $\alpha$ are $\alpha_{1}=0.16$ $\pm 0.01, \alpha_{2}=0.14 \pm 0.01$, and $\alpha_{3}=0.35 \pm 0.01$. The error bars on the points indicate the range of possible extrapolation errors estimated by using maximum and minimum slopes compatible with the data in the extrapolation. In the present case since $\rho(0) \propto L^{-0.1072}$, we have $\bar{K}_{l} \propto L^{-\tau_{l}}$ [or $\left.\rho(0)^{T_{i}}\right]$, where $\tau_{1}=0.27\left(T_{1}=2.5\right), \tau_{2}=0.25\left(T_{2}=2.3\right)$, and $\tau_{3}=0.46\left(T_{3}=4.3\right)$. It is seen that whereas $\tau_{1} \approx \tau_{2}$ within the errors of calculation, $\tau_{3}$ definitely differs from $\tau_{1}$ and $\tau_{2}$. Calculations have also been performed using different ratios of $\mu / \lambda$. The exponents obtained are the same, demonstrating the independence of the exponent values from material properties. It should be noted that as a consequence of $\bar{K}_{1}$ 

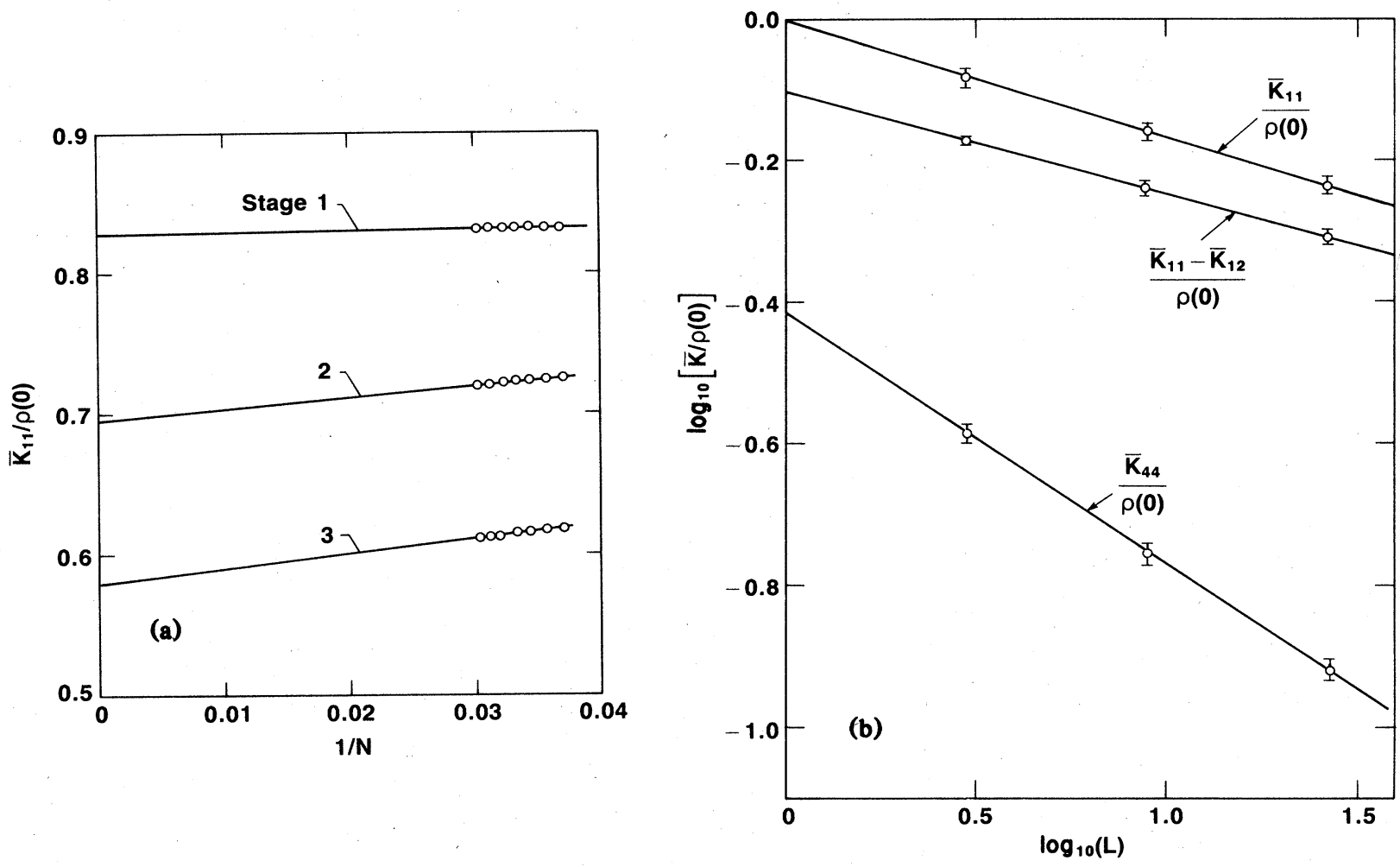

FIG. 2. (a) Variation of the moduli as a function of $N$.

(b) Variation of the carpet moduli as a function of sample size $L$.

and $\bar{K}_{3}$ scaling differently, the bulk modulus, or other combinations of $\bar{K}_{1}$ and $\bar{K}_{3}$, does not exhibit scaling behavior as a function of $L$ in the initial stages. Also, the ratio of $v_{s}=\left[\bar{K}_{44} / \rho(0)\right]^{1 / 2}$ to $v_{p}=\left[\bar{K}_{11} / \rho(0)\right]^{1 / 2}$ approaches zero as $L \rightarrow \infty$.

There are usually corrections to scaling behaviors which tend to zero as $L \rightarrow \infty$. In the present case, the corrections are surprisingly small as evidenced by (1) the excellent power-law behavior exhibited by the initial three stages and (2) the iterated cases, such as substituting stage 1 result back into stage 1 (in that case the solid is locally cubic) or substituting moduli of stage 1 into stage 2 , all yield results almost indistinguishable from the directly calculated stage 2 or the stage 3 results ( $<3 \%$ difference). This latter fact shows that for the Sierpinski carpet, the scaling behavior of the three moduli begins almost immediately at stages 1 and 2.

Since our results differ qualitatively from those of previous discrete models, it is interesting to speculate on the origin of the discrepancy. One possibility is that the features observed in the present work are special to the Sierpinski carpet and its associated symmetry, suggesting that the number of distinct elastic scaling behaviors is highly symmetry dependent. Another contributing factor may be the breakdown of analogy between the discrete and continuum elasticity ${ }^{7}$ for fractal and percolation cluster models. It is well known that while discrete models can exhibit classical elasticity behavior in the limit when the number of springs and nodes are large, there are nevertheless, clear differences between the characteristics of continuum elasticity and those of a single (or a few) spring(s). In view of the importance of single-spring connections in discrete, fractal, or percolation cluster models, it would not be surprising that such differences could account for the different scaling behaviors. It is proposed that a careful experimental study of twodimensional and three-dimensional elastic fractal structures could shed illuminating insights on these issues.

We wish to thank Y. Kantor and T. Witten for useful discussions.
"Permanent address: Department of Physics, Fudan University, Shanghai, People's Republic of China.

1S. Feng and P. N. Sen, Phys. Rev. Lett. 52, 216 (1984).

${ }^{2}$ Y. Kantor and I. Webman, Phys. Rev. Lett. 52, 1891 (1984).

${ }^{3}$ D. J. Bergman and Y. Kantor, Phys. Rev. Lett. 53, 511 (1984).

${ }^{4}$ B. B. Mandelbrot, Fractals, Form, Chance, and Dimension (Freeman,
San Francisco, 1977), p. 164.

${ }^{5}$ See, for example, W. L. Pilant, Elastic Waves in the Earth (Elsevier, New York, 1979), p. 34.

${ }^{6} R$. Tao and P. Sheng, J. Acoust. Soc. Am. (to be published).

${ }^{7}$ Y. Kantor (private communication). 